\title{
Application of the Zhe Yin's Gene Inherits Law
}

\author{
Zhe Yin',2, Yunfei Guo1 \\ ${ }^{1}$ Mathematics Department, Yanbian University, Yanji, China \\ ${ }^{2}$ Department of Information Management, Peking University, Beijing, China \\ Email: yinzhe@ybu.edu.cn
}

Received 16 November 2014; revised 5 December 2014; accepted 18 December 2014

Copyright (C) 2014 by authors and Scientific Research Publishing Inc.

This work is licensed under the Creative Commons Attribution International License (CC BY).

http://creativecommons.org/licenses/by/4.0/

(c) (i) Open Access

\section{Abstract}

Several results are provided using gene inherits law in another article [1], making use of the theory of algebra in mathematics, according to which we derive, which will give some reference value to genetics.

\section{Keywords}

\section{Inherits Law, Memory Inheritance, Memory Copy}

\section{Introduction}

Right now, many laws in genetics can be derived from the statistical law of genetic results. There is rather little research on principle. Can a reasonable estimate of the genetic results be presented from the perspective of principle? The answer is yes. Zhe Yin's gene inherits law in reference [1] will be introduced first.

\section{Two Lemmas}

Genetic behavior is divided into dominant inheritance and recessive inheritance. Assume that $D$ represents for dominant inheritance factor, and $d$ represents for recessive inheritance factor; there are two cases among inheritance factors, that is, dependent and independent; independent refers to there is cross effect between $D$ and $d$, that is, $D d$ is also one inheritance result.

The possible results may be $D$ or $d$ when inheritance factors of male parent and female parent are independent.

The possible results may be $D, d,(D d)^{+},(D d)^{-}, D(D d)^{+}, d(D d)^{+}, D(D d)^{-},(D d)^{-} d$ when inheritance factors of male parent and female parent are dependent. 
Binomial equation formula:

$$
(D+d)^{n}=\sum_{i=0}^{n} C_{n}^{i} D^{i} d^{n-i},
$$

where

$$
C_{n}^{i}=\frac{n !}{i !(n-i) !} .
$$

Coefficients of the odd and even items in the expansion are equal.

Expansion $(D+d)^{n}=\sum_{i=0}^{n} C_{n}^{i} D^{i} d^{n-i}$ is just all of the possible results of the $n-1$ th generation.

Lemma 1. When male parent and female meet dominant inheritance, inheritance factors fit such addition principle 1:

$$
\begin{aligned}
& D \oplus D=D ; \\
& D \oplus d=D ; \\
& d \oplus D=D ; \\
& d \oplus d=d .
\end{aligned}
$$

Lemma 2. When male parent and female meet recessive inheritance, inheritance factors fit such addition principle 2:

$$
\begin{aligned}
& D \oplus D=d ; \\
& D \oplus d=D ; \\
& d \oplus D=D ; \\
& d \oplus d=d .
\end{aligned}
$$

\section{Zhe Yin's Gene Inherits Law}

Zhe Yin's gene inherits law:

1) All of the possible inheritance results of the $n-1$ th generation fit such expansion:

$$
(D+d)^{n}=\sum_{i=0}^{n} C_{n}^{i} D^{i} d^{n-i}=C_{n}^{0} D^{n}+C_{n}^{1} D^{n-1} d+C_{n}^{2} D^{n-2} d^{2}+\cdots+C_{n}^{n} d^{n}
$$

where

$$
C_{n}^{i}=\frac{n !}{i !(n-i) !}
$$

2) Inheritance factors meet distributive law (separate regularity);

3) Inheritance factors meet associative law (law of independent assortment);

4) Dominant inheritance meets addition principle 1;

5) Recessive inheritance meets addition principle 2;

6) When $D$ and $d$ are independent, possible results are $D$ or $d$;

7) When $D$ and $d$ are dependent, $D d$ will be regarded as inheritance factor which also have dominant inheritance $(D d)^{+}$and recessive inheritance $(D d)^{-}$(linkage inheritance), and possible results are: $D, d$, $(D d)^{+},(D d)^{-}, D(D d)^{+}, d(D d)^{+}, D(D d)^{-},(D d)^{-} d$.

Note: when $n=2$, this inheritance law is just Mendel's law.

\section{Application of Zhe Yin's Gene Inherits Law}

\subsection{Application 1: Subtraction Principle of Genetic (Inference of Zhe Yin's Gene Inherits Law)}

According to the theory of algebra in mathematics. (According to the theory of algebra, addition and sutration in 
Lemma 1 and Lemma 2 are equivalent in Zhe Yin's gene inherits law) if addition principle in Lemma 1 and Lemma 2 is right, subtraction principle in Lemma 1 and Lemma 2 will be also met. That is,

Lemma 1. When male parent and female meet dominant inheritance, inheritance factors fit suchsubtraction principle 1:

$$
\begin{aligned}
& D \odot D=D ; \\
& D \odot d=D ; \\
& d \odot D=D ; \\
& d \odot d=d .
\end{aligned}
$$

Lemma 2. When male parent and female meet dominant inheritance, inheritance factors fit such subtraction principle 2:

$$
\begin{aligned}
& D \odot D=d ; \\
& D \odot d=D ; \\
& d \odot D=D ; \\
& d \odot d=d .
\end{aligned}
$$

Addition and subtraction in math and algebra are equivalent, but its significance in biology is very large. Addition represents the result of the integration, subtraction represents differences or changes in content. This is mainly reflected that the living things gain and memory only changed information to outside information, but gain and memory same information to non-continuous ones, all the way to genetic. Not only humans, animal information of vision, taste, smell, and so on have also taken changing nature of memory storage.

Biological evolution is gene mutation with the environmental impact so dominant inheritance refers to biological evolution. Mutation evolutionary characteristics changed at three times speed compared to the previous original features (original features before the evolutionary genetic increment $=0$, genetic increment which has the gene mutation evolutionary characteristics = three times); characteristic of recessive inheritance refers to keep 50\% evolution before, keep 50\% after mutation evolutionary characteristics (genetic process was changed on the second process, preserving $50 \%$ in fact equals no change). It is worth mentioning that with the impact of environment, dominant and recessive hereditary interchangeable. For a single organism, overt or tissue tested positive for the virus were negative, occult viral or cellular organization. Normal number of latent virus in human reproduction cells without incremental, but the volume increased 1 time (according to coefficient ratio in Zhe Yin's gene inherits law).

\subsection{Application 2: The Dynamics and Monotonic of Genetic}

Genetic process is a dynamic process. According to Zhe Yin's gene inherits law, which is that continuous evolution process of bio at three times speed, is also that living beings remain $100 \%$ trait genes in the process. Dominant hereditary represents of biological evolution (environmental influences), recessive hereditary represents of biological organisms maintaining the original characteristics. With the impact of environment, dominant and recessive hereditary interchange each other. Genetic variation is equivalent to cell variation. If different paternity rate $=Y$, identification of children $=N, Y=F(n)$, when $N$ becomes large, $Y$ increases monotonically. When the value of $N$ is equal, parentage living in different environments with different rate of $Y$-value is greater than the same conditions paternity rates of different $Y$ values.

For example, cancer cells is the biological evolution of dominant process, AIDS was bio-conservative (degradation) of recessive processes. Low immunity sharply conservative phenomenon of biological degradation phenomena, is very likely to be the next of kin at the intersection of product. It is worth mentioning that without distinction as to race, looks like many of them are mentally handicapped groups striking similarities have a relationship with inbreeding. Inbreeding is the most effective way of returning to the ancient.

\subsection{Application 3: Twice Property of Genetics}

Zhe Yin's gene inherits law is the one about individual gene, complete thought is included of the collective of gene, which is inheritance that is composed of gene strand by media (such as memory piece). Union of the male 
germ cell and female germ cell nuclei of genetic inheritance can be identified for the first inheritance, female genetic memory (memory copy of the parent to the young) can be identified as the second genetic or epigenetic inheritance. The first inheritance contains the basic genetic information (part of evolutionary information is not included), the second inheritance contains part of evolutionary information, through the copying and passing brain's memory information.

Human or animal instinct actually does not exist, is copying and inheritance from a generation ago, previous generations of ancestors (hereditary for the second time). Human's dreaming phenomenon are previous generations of ancestral memory contents and mix back to the recent memory. After copying the creature's young brain gene mutations identified as genetic variability for the second time.

\subsection{Application 4: Rules of Blood Type Inheritance}

A-type and B-type blood are dominant inheritance, AB-type is a double dominant genetic, and O-type is recessive. According to Zhe Yin's gene inherits law, we have:

Blood type of parents:

$\mathrm{A}$ and $\mathrm{A}:(\mathrm{A}+\mathrm{O}) \oplus(\mathrm{A}+\mathrm{O})=\mathrm{AA}+2 \mathrm{AO}+\mathrm{OO}=3 \mathrm{~A}+\mathrm{O} \rightarrow 3: 1$;

$\mathrm{A}$ and $\mathrm{B}:(\mathrm{A}+\mathrm{O}) \oplus(\mathrm{B}+\mathrm{O})=\mathrm{AB}+\mathrm{AO}+\mathrm{BO}+\mathrm{OO}=\mathrm{AB}+\mathrm{A}+\mathrm{B}+\mathrm{O} \rightarrow 1: 1: 1: 1$

$\mathrm{A}$ and $\mathrm{O}:(\mathrm{A}+\mathrm{O}) \oplus(\mathrm{O}+\mathrm{O})=\mathrm{AO}+\mathrm{AO}+\mathrm{OO}+\mathrm{OO}=2 \mathrm{~A}+2 \mathrm{O} \rightarrow 1: 1$

$\mathrm{A}$ and $\mathrm{AB}:(\mathrm{A}+\mathrm{O}) \oplus(\mathrm{A}+\mathrm{B})=\mathrm{AA}+\mathrm{AB}+\mathrm{AO}+\mathrm{BO}=2 \mathrm{~A}+\mathrm{AB}+\mathrm{B} \rightarrow 2: 1: 1$

$\mathrm{B}$ and $\mathrm{B}:(\mathrm{B}+\mathrm{O})(\mathrm{B}+\mathrm{O})=\mathrm{BB}+2 \mathrm{BO}+\mathrm{OO}=3 \mathrm{~B}+\mathrm{O} \rightarrow 3: 1$;

$\mathrm{B}$ and $\mathrm{AB}:(\mathrm{B}+\mathrm{O}) \oplus(\mathrm{A}+\mathrm{B})=\mathrm{AB}+\mathrm{BB}+\mathrm{AO}+\mathrm{BO}=\mathrm{AB}+2 \mathrm{~B}+\mathrm{A} \rightarrow 1: 2: 1$;

$\mathrm{O}$ and $\mathrm{O}:(\mathrm{O}+\mathrm{O}) \oplus(\mathrm{O}+\mathrm{O})=4 \mathrm{O} \rightarrow 1$;

$\mathrm{O}$ and $\mathrm{AB}:(\mathrm{O}+\mathrm{O}) \oplus(\mathrm{A}+\mathrm{B})=2 \mathrm{AO}+2 \mathrm{BO}=2 \mathrm{~A}+2 \mathrm{~B} \rightarrow 1: 1$;

$\mathrm{B}$ and $\mathrm{O}:(\mathrm{B}+\mathrm{O}) \oplus(\mathrm{O}+\mathrm{O})=\mathrm{BO}+\mathrm{BO}+\mathrm{OO}+\mathrm{OO}=2 \mathrm{~B}+2 \mathrm{O} \rightarrow 1: 1$;

$A B$ and $A B:(A+B) \oplus(A+B)=A A+A B+A B+B B=A+2 A B+B \rightarrow 1: 2: 1$. See blood type inheritance table for details [2] (Table 1$)$.

\subsection{Application 5: Atavism}

When the hereditary and sex-linked recessive traits are met, according to Zhe Yin's gene inherits law, according to corollary 5 and 6 in reference [1], atavism will be took place. That is, even-numbered generation inherits but odd-numbered one doesn't inherit, such as hemophilia, red-green colorblind, pseudohypertrophic muscular dystrophy and-6-glucose dehydrogenate deficiency, and so on.

\section{Table 1. Blood type inheritance table.}

\begin{tabular}{ccc}
\hline Blood type of parents & Blood type of children & Ratio \\
\hline A and A & A, O & A:O $=3: 1$ \\
A and B & AB, A, B, O & A:B:O $=1: 1: 1: 1$ \\
A and $O$ & A, O & A:O $=1: 1$ \\
A and AB & A, AB, B $:$ B:B $=2: 1: 1$ \\
B and B & B, O & B:O $=3: 1$ \\
B and AB & AB, B, A & AB:B:A $=1: 2: 1$ \\
O and O & O & O $=1$ \\
O and AB & A, B & A:B $=1: 1$ \\
B and $O$ & B, O & B:O $=1: 1$ \\
AB and AB & A, AB, B & A:AB:B $=1: 2: 1$ \\
\hline
\end{tabular}

Blood group inheritance and ratio with a simple calculation in a similar way. AB type blood and A type or B type blood occur gene mutations linked, which can create special blood type. 


\section{Conclusion}

Based on the Zhe Yin's gene inherits law using the principles of algebra, feasible ideas about biology have been clearly provided. According to Zhe Yin's gene inherits law, inheritance and evolutionary process has been quantized, which will play a references role in future.

\section{References}

[1] Yin, Z. (2012) The Law of Genetic Inheritance. Open Journal of Genetics, 2, 47-50. http://dx.doi.org/10.4236/ojgen.2012.21005

[2] Yin, Z. and Guo, Y.F. (2012) Principle of Blood Group Inheritance and Calculation of Its Ratio. Pure and Applied Biology, 1, 16-17. 
Scientific Research Publishing (SCIRP) is one of the largest Open Access journal publishers. It is currently publishing more than 200 open access, online, peer-reviewed journals covering a wide range of academic disciplines. SCIRP serves the worldwide academic communities and contributes to the progress and application of science with its publication.

Other selected journals from SCIRP are listed as below. Submit your manuscript to us via either submit@scirp.org or Online Submission Portal.
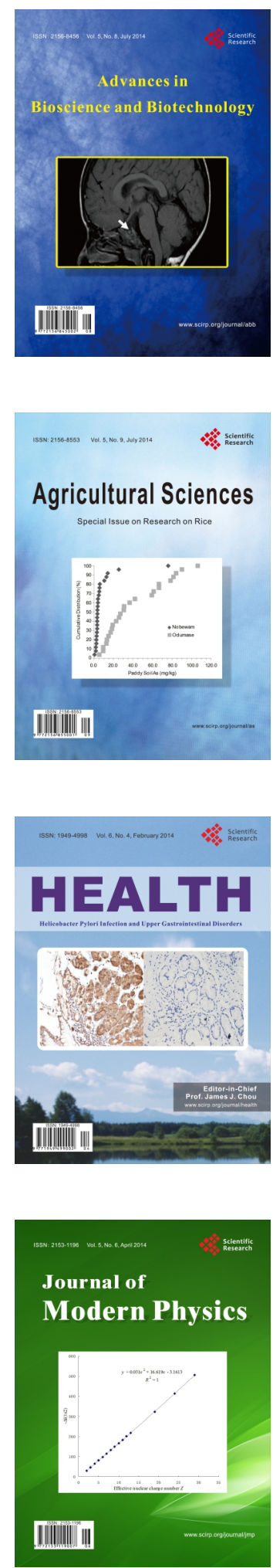
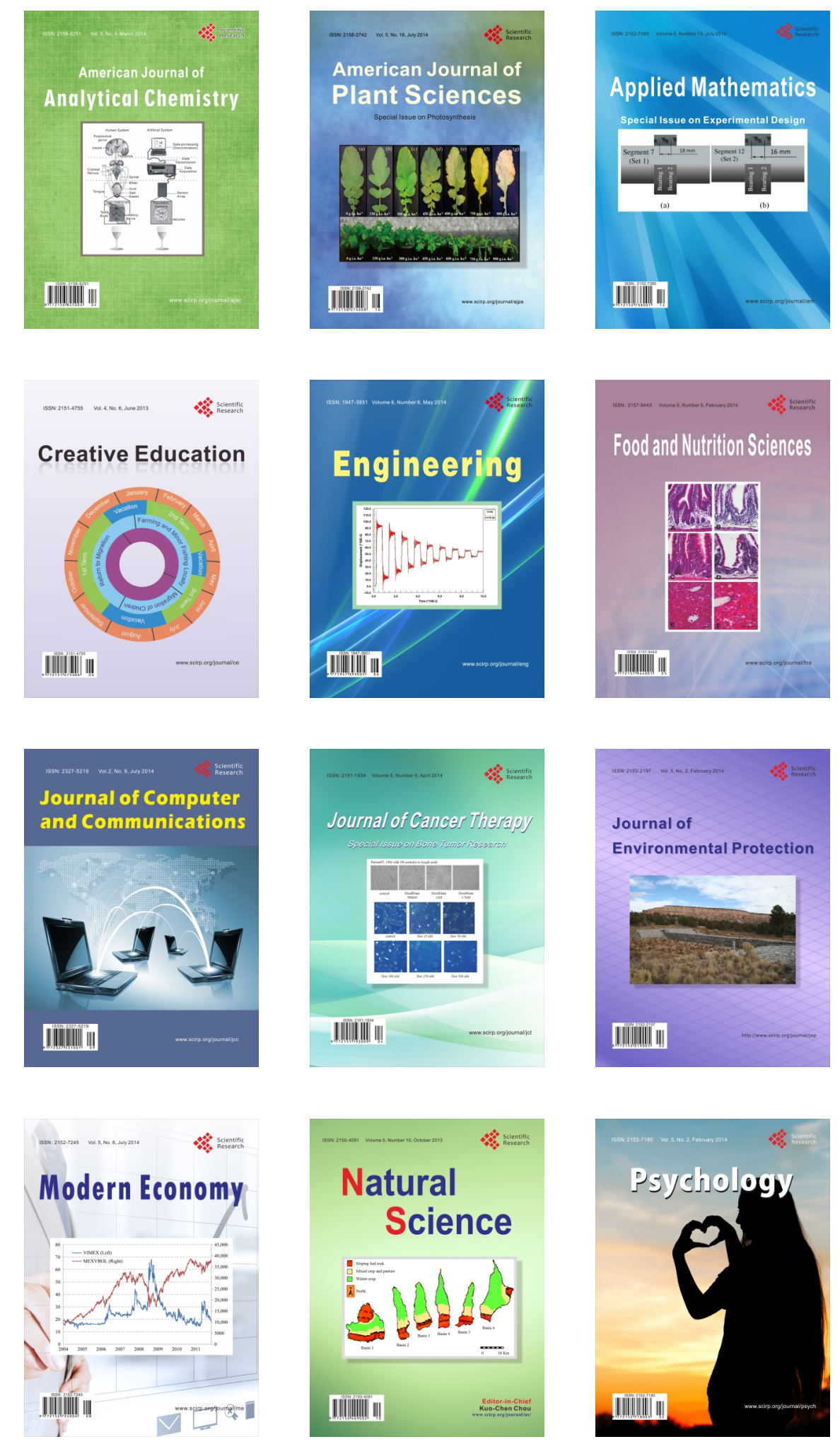